\title{
Filigrane
}

Écoutes psychanalytiques

\section{Présentation du numéro}

\section{Sophie Gilbert}

Volume 28, numéro 1, 2019

URI : https://id.erudit.org/iderudit/1064592ar

DOI : https://doi.org/10.7202/1064592ar

Aller au sommaire du numéro

\section{Éditeur(s)}

Santé mentale et société

\section{ISSN}

1192-1412 (imprimé)

1911-4656 (numérique)

Découvrir la revue

Citer ce document

Gilbert, S. (2019). Présentation du numéro. Filigrane, 28(1), 5-8.

https://doi.org/10.7202/1064592ar d'utilisation que vous pouvez consulter en ligne.

https://apropos.erudit.org/fr/usagers/politique-dutilisation/ 


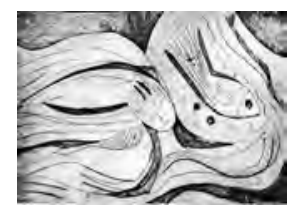

\title{
Présentation du numéro
}

\author{
Sophie Gilbert
}

i pour les élèves du primaire, l'apprentissage de la langue française culin, avant même d'avoir franchi le seuil de l'école secondaire, nos jeunes tendent désormais à se situer dans une multiplication des identités et orientations sexuelles. La clinique adolescente le confirme: aucun flottement n'est toléré, et ces définitions précèdent souvent de loin l'abord et parfois même, la simple fantaisie de l'acte sexuel. On se demande s'il n'y a pas ici ratage d'une double rencontre: la rencontre de soi comme celle de l'altérité quelle qu'elle soit, mais surtout, la possibilité de supporter le non-savoir qui éventuellement aboutira à une identité, si ce n'est définitive, à tout le moins confortable. Et si cette obligation à se situer, à circonscrire le genre, aussi fertile ait pu être son élaboration première, tendait à refermer une question qu'on espérait désormais ouverte, à stigmatiser davantage qu'à relier?

Tout se passe comme si le simple mot genre, lorsqu'il signifie un au-delà de la dualité qu'on lui connaissait jusqu'alors, crée autant de remous chez nos jeunes que de malaise (malêtre?) chez les cliniciens.

Étymologiquement, le mot genre réfere à une catégorie. Du reste, il est intéressant de noter que son usage dans la lange orale, au Québec, est venu ponctuer pour toute une génération (ou même plusieurs) les phrases des ados, des jeunes et des «moins jeunes», de façon à signifier une modulation du propos précédent - comme si l'on disait constamment, à la fin d'une assertion peu importe laquelle: «Ce n'est pas tout à fait ça.» Ce pas tout à fait, associé à l'étymologie, rappelle fort justement les limites de toute tentative de catégorisation. En d'autres termes, cet entendement du «genre» met en exergue ce qui fait (et fera sans doute) toujours défaut dans la question identitaire abordée sous l'angle d'une classification, ce en quoi chacun est d'abord «un» et jamais tout à fait comme l'autre. Ici, la référence au «trait unaire» lacanien est bien sûr inspirante, dans sa façon d'articuler l'unicité identitaire avec la différence, l'altérité. Parler de genre, c'est donc d'emblée introduire la question de l'identité, voire des identités, mais aussi de l'autre, de l'altérité, et de la différence. 
Intitulé «Identités². Qui suis-je?», le colloque de l'automne 2018 de la revue Filigrane a proposé une rencontre entre différents cliniciens, réunis par leur intérêt commun pour la remise en question actuelle de la notion d'identité sexuelle. L'expérience de ce colloque entrera dans les annales de la revue pour plusieurs raisons. L'une d'elle tient en la difficulté à initier un véritable dialogue, des échanges heuristiques à partir des controversées thématiques de l'identité sexuelle et du genre. À la lumière du propos précédent, l'on pourrait mettre en cause la propension possiblement vouée à l'échec de catégoriser les identités. Plus encore, en référence à l'expérience, relevée ci-dessus, de la clinique adolescente, l'on peut se demander à quel point cette tentative de catégoriser, malgré le désir de soutenir l'ouverture à tout un chacun, a un effet pervers de fermeture (de «forclusion»?) de la question identitaire par le point final qui en découle. Ces arguments pourraient sans doute expliquer, théoriquement, l'impasse dans laquelle se sont retrouvés, malgré le bon vouloir des organisateurs, nos cliniciens invités. Chacun a su parler du genre en référence à son allégeance théorique et son expérience clinique; toutefois, peu de résonances ont su être relevées entre les entendements (forcément sous-tendus par des catégories explicites ou implicites) proposés, et chaque élaboration, aussi riche fut-elle, semble avoir été ponctuée d'un point final.

La psychanalyse, on l'a bien vu les dernières années par une multiplication du nombre de publications sur ce thème, a bien voulu si ce n'est intégrer le genre en tant que concept, du moins tenter de l'approcher avec des concepts fondamentaux dont bien sûr la bisexualité psychique demeure au premier plan. Reste que lorsqu'il est question de mettre au travail ce thème, en lien avec certaines expériences cliniques, la rencontre de l'autre demeure difficile, quoique désirée ${ }^{1}$.

Plus précisément, dans le cadre du colloque de la revue Filigrane, l'inconscient s'est heurté, de plein fouet dirais-je, au roc de La Réalité. A priori, il semble que tous les participants, conférenciers ou auditeurs, se référaient à un même paradigme, constructiviste, et à la notion de réalité psychique, voire même, à l'existence de l'inconscient. Pourtant, l'on pourrait penser que l'inconscient de ces hommes ou femmes qui souhaitent explorer une autre image de soi, un soi plus conforme à leur intériorité, ait à certains moments été éludé. Ce faisant, les concepts de conflits psychiques, de transmission générationnelle, pour ne nommer que ceux-là, n'étaient pas toujours au rendez-vous: l'individu était considéré à son niveau conscient, selon «son point de vue» (aconflictuel?). Si l'autre était interpellé, c'était 
essentiellement pour signifier son manque de tolérance à la différence. Un argument, soit dit en passant, fondamental en ce qui concerne les personnes trans et la stigmatisation dont elles sont victimes!

D'un autre côté, il semble que l'analyse en profondeur, le point de vue intrapsychique, rende périlleux le dialogue avec une réalité particulièrement insistante chez les personnes trans car liée à un prégnant malêtre. Peut-on parfois, sous prétexte d'une compréhension exhaustive des enjeux intrapsychiques, représenter malgré soi cet autre craint, car insuffisamment sensible à la souffrance au quotidien, fut-elle narcissique et identitaire ${ }^{2}$ ?

Un autre niveau d'analyse de cette non-rencontre nous amène à poser les questions suivantes. Serait-il possible que plus l'on arrive à se distancier (défensivement ou après analyse personnelle?) des enjeux identitaires fondamentaux incarnés par les personnes trans, plus l'on peut soutenir une théorisation de la dynamique psychique de (et pas toujours avec) ceux-ci? À l'inverse, plus on s'identifierait à cette souffrance identitaire, plus l'on connecterait avec ce malêtre (profondément humain, l'identité n'est-elle pas le travail d'une vie?), et plus l'on chercherait à répondre à cette souffrance qui rejoint l'humain dans ses plus profonds soubassements, en interpellant non seulement le sujet souffrant mais également le social auquel il est durement confronté?

Jacques André nous rappelle combien l'accession au langage a transformé l'animal en nous. Mais il y a un prix à payer à cette désignation, le genre, toujours imparfaite, à la fois teintée d'enjeux sociopolitiques, de la culture, et de la complexité du développement psychique du sujet. Les cliniciens-auteurs qui ont répondu à notre argumentaire dans ces deux numéros thématiques de Filigrane se sont unanimement, quoique de façon distincte, montrés sensibles à cette complexité inhérente à la notion de genre, mais aussi à la diversité à la fois phénoménologique et conceptuelle de la notion plurielle d'identité. Chacun pourra se demander, au fil de sa lecture, comment ouvrir un véritable dialogue entre considérations psychiques et socioculturelles, entre le sujet et l'altérité, entre dynamique inconsciente et vécu subjectif.

Le dossier thématique s'inaugure par une introduction proposée par Alexandre L'Archevêque (membre du comité de rédaction de la revue Filigrane), qui a servi d'argumentaire à l'ensemble de nos auteurs. La suite de cette première partie du dossier fait place à une majorité des conférences 
proposées lors du colloque. La conférence d'ouverture signée par Jacques André aborde d'un point de vue théorique le caractère distinct de la perspective psychanalytique sur le genre, notamment de par son incontournable référence à l'inconscient. Le généreux commentaire proposé par Dominique Scarfone (discutant de cette conférence) est ensuite présenté, permettant d'affiner la réflexion sur certains thèmes fondamentaux tels le sexuel infantile et le déterminisme inconscient. Le texte de la seconde conférence de Jacques André aborde ensuite de front le concept de bisexualité psychique à la lumière de vignettes cliniques. Pour clore cette première partie du dossier, la riche complémentarité offerte par la perspective phénoménologique est introduite par Denise Medico, clinicienne et chercheure d'expérience auprès des populations trans.

La rubrique «Hétéros» amène les lecteurs sur un autre terrain, celui des violences sexuelles à l'adolescence. Pascal Roman illustre son propos sur les enjeux narcissiques et identitaires inhérents à cette violence par une vignette clinique étoffée.

Puis, dans la rubrique «Échos», deux courts textes rédigés par Domique Scarfone revisitent de façon originale deux concepts phares pour la psychanalyse: l'inconscient et le statut du savoir.

En terminant, ce numéro fait place à trois articles rédigés par des professeurs de la section psychodynamique du Département de psychologie de l'Université du Québec à Montréal. Ces articles donnent un aperçu de la diversité des points de vue et des travaux d'orientation psychanalytique de ce département qui se démarque par un important contingent de professeurs de cette allégeance. Louis Brunet formalise ici le travail d'une réflexion extensive sur le statut de la psychothérapie d'orientation psychanalytique, Irène Krymko-Bleton aborde la méthodologie de recherche qu'elle a développée dans une perspective psychanalytique inspirée de la linguistique, et finalement, Marie Hazan lève le voile sur une facette méconnue de la vie d'Anna Freud.

\section{Notes}

1. À noter qu'à l'image du colloque de la revue Filigrane, le Congrès des psychanalystes de langue française de 2019 faisait place également à d'autres disciplines.

2. J'utilise à dessein ce terme ici, afin de désigner le lieu où s'inscrit le malêtre dont il est question. Ce faisant, l'on peut entrevoir de nouveau une source de malentendu. De fait, la souffrance narcissique-identitaire a un entendement bien précis en psychanalyse, notamment à la suite des travaux de Roussillon. Comment peut-on témoigner de ce lien, sans plaquer une compréhension pré-formatée sur l'expérience des personnes trans? 